\title{
Spatially Resolved Vibrational Electron Energy-loss Spectroscopy Across an Abrupt $\mathrm{SiO}_{2} / \mathrm{Si}$ Interface
}

\author{
Kartik Venkatraman ${ }^{1}$, Katia March $^{2}$, Peter Rez ${ }^{3}$ and Peter A. Crozier ${ }^{1}$ \\ 1. SEMTE, Arizona State University, Tempe, Arizona, USA. \\ 2. Eyring Materials Center, Arizona State University, Tempe, Arizona, USA. \\ 3. Department of Physics, Arizona State University, Tempe, Arizona, USA.
}

The detection of vibrational excitations at high spatial resolution with monochromated vibrational electron energy-loss spectroscopy (EELS) can be used to perform local materials characterization on complex geometries. To develop a comprehensive understanding of this technique so that it can be applied to complex materials systems, we need to perform experiments on relatively simple model systems and compare experiment with theory. Recent work on the influence of an abrupt $\mathrm{SiO}_{2} / \mathrm{Si}$ interface on the spatially resolved Si-O bond stretch vibrational signal showed a signal in $\mathrm{SiO}_{2} 200 \mathrm{~nm}$ from the interface due to the long-range Coulomb interaction. At the interface the integrated signal intensity drops to zero. Nanometer spatial resolution is achievable while selecting the interfacial vibrational stretch signal as the electron beam is scanned across the interface. It is also shown that surface coupling must be considered for typical TEM sample thicknesses. Moreover, the energy-loss spectra in $\mathrm{SiO}_{2}$ can be interpreted using the non-relativistic dielectric theory, while relativistic effects must be considered in $\mathrm{Si}$ [1]. In this paper, we explore the experimental spatial variation of the Si-O vibrational modes as the electron probe is scanned across the interface. The experimental spectra are interpreted in terms of the relativistic dielectric theory [2].

A $3 \mu \mathrm{m}$ layer of $\mathrm{SiO}_{2}$ on a $\mathrm{Si}$ wafer was prepared for STEM EELS analysis by lifting out a focused ion beam (FIB) sample using a Nova 200 NanoLab (FEI) FIB. A NION UltraSTEM 100 aberrationcorrected electron microscope equipped with a monochromator was used to perform EELS linescans across the $\mathrm{SiO}_{2} / \mathrm{Si}$ interface. The microscope was operated at $60 \mathrm{kV}$, with probe convergence and collection semi-angles of 30 and 12 mrad respectively. The experimental EELS energy-resolution was $15 \mathrm{meV}$. Background subtraction and signal integration were performed using the Gatan Microscopy Suite.

Figure 1a is a bright-field (BF) STEM image of the $\mathrm{SiO}_{2} / \mathrm{Si}$ interface that shows the position of the interface and the direction of the linescan. A typical background subtracted vibrational energy-loss spectrum observed when the electron beam is positioned in $\mathrm{SiO}_{2}$ far away from the interface is shown in Figure 1b. The energy-loss spectrum shows three peaks at 58, 98 and $144 \mathrm{meV}$ when the beam is in $\mathrm{SiO}_{2}$, which correspond to the thin-film Si-O bond-rocking, bond-bending and bond-stretching vibrational signals respectively [3].

Figure 2a shows the experimental spatial variation of the $58 \mathrm{meV} \mathrm{Si-O} \mathrm{bond-rocking} \mathrm{signal} \mathrm{overlaid} \mathrm{on}$ the spatial variation of the $144 \mathrm{meV}$ signal in $\mathrm{SiO}_{2}$. The two profiles look very similar which suggests that a begrenzungs type of effect causes the $58 \mathrm{meV}$ integrated signal intensity to drop from its maximum value far away from the interface to zero at the interface [1]. Figure $2 \mathrm{~b}$ shows the variation in surface contribution with change in thin-film thickness, calculated using Kröger's formula [2]. If the 
sample thickness is of the order of $\mathrm{v} / \omega$ ( $\mathrm{v}$ is the electron velocity, $\omega$ is the energy-loss frequency), a significant surface contribution is observed in the energy-loss spectra [4]. For $60 \mathrm{kV}$ accelerating voltage, $\mathrm{v} / \omega$ is $\sim 600 \mathrm{~nm}$ for $144 \mathrm{meV}$ and $\sim 1500 \mathrm{~nm}$ for $58 \mathrm{meV}$. This suggests that while there is competing contribution from bulk and surface for the $144 \mathrm{meV}$ signal, only the surface contribution is observed for the $58 \mathrm{meV}$ signal. Experimental spatial variation profiles for the bond-rocking signal as the probe moves into $\mathrm{Si}$ and detailed simulations to interpret the experimental energy-loss spectra in $\mathrm{Si}$ will be presented [5].

\section{References:}

[1] K. Venkatraman et al, Microscopy (2018), p. 1-10, doi: 10.1093/jmicro/dfy003.

[2] E. Kroger, Zeitschrift für Physik 216 (1968), p. 115.

[3] M. Hass, J. Phys. Chem. Solids 31 (1970), p. 415.

[4] R.F. Egerton, Electron Energy-Loss Spectroscopy in the Electron Microscope, $2^{\text {nd }}$ ed. (Plenum Press, New York, 1996).

[5] The support from National Science Foundation CHE-1508667 and the use of (S)TEM at Eyring Materials Center at Arizona State University is gratefully acknowledged.
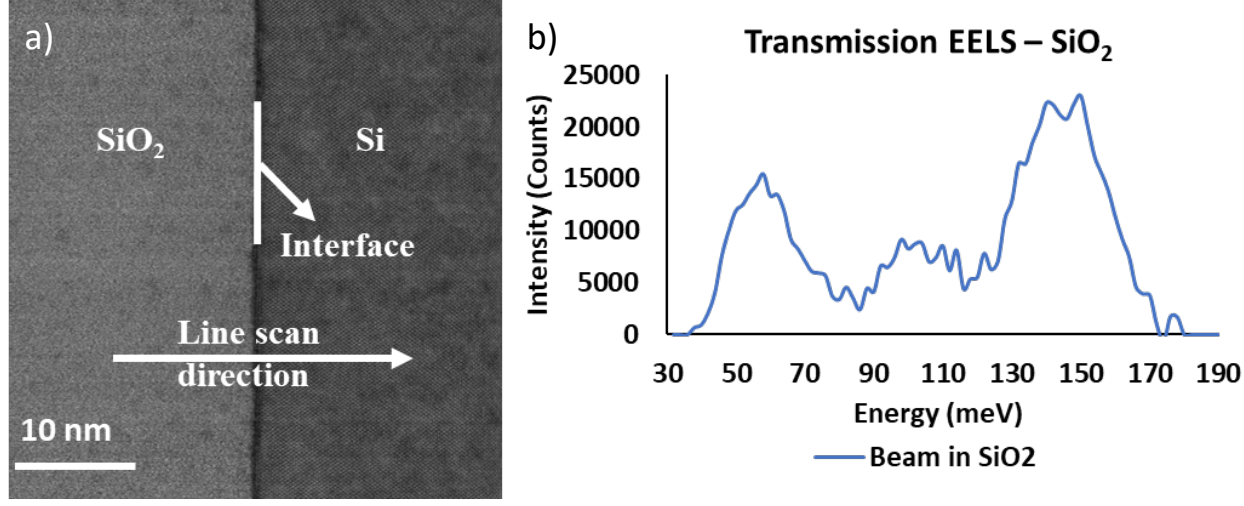

Figure 1. a) BF STEM image of the $\mathrm{SiO}_{2} / \mathrm{Si}$ interface showing the direction of linescan. b) Typical experimental vibrational spectra when the beam is positioned far away $(\sim 400 \mathrm{~nm})$ from the interface and at the interface.
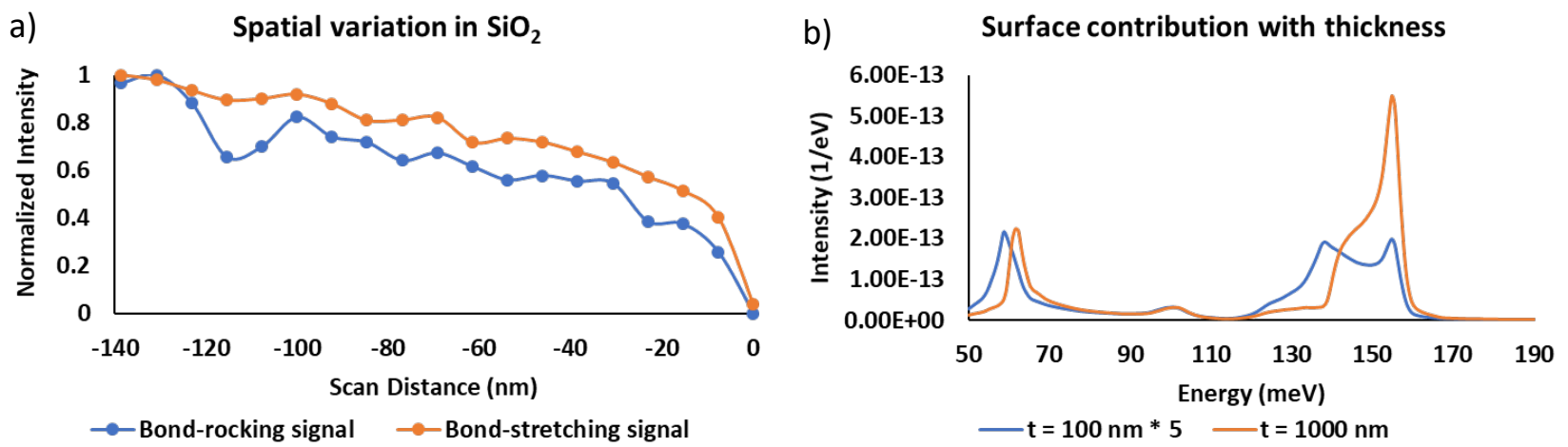

Figure 2. a) Experimental spatial variation of the $58 \mathrm{meV}$ bond-rocking signal overlaid on that of the $144 \mathrm{meV}$ bond-stretching signal in $\mathrm{SiO}_{2}$. b) Difference in the variation of surface contribution with thickness for the 58 $\mathrm{meV}$ and $144 \mathrm{meV}$ signals - calculated using Kröger's formula [2]. 